\title{
CEO advisory
}

\section{Leadership and strategy in the news}

\section{Craig Henry}

\author{
Craig Henry, Strategy \& Leadership's \\ intrepid media explorer, collected \\ these examples of novel strategic \\ management concepts and \\ leadership practices and impending \\ environmental discontinuity from \\ various news media. A marketing and \\ strategy consultant based in Carlisle, \\ Pennsylvania, he welcomes your \\ contributions and suggestions \\ (craig_henry@centurylink.net).
}

\author{
Of strategies and strategists
}

\section{Making strategy: Understanding when and how to argue}

Netflix has driven stunning transformations in the media landscape in part through its success at encouraging its leaders to debate ideas frankly and its willingness to empower them to take risks without waiting for an annual strategy planning process. It is no surprise that CEO Reed Hastings views working from home as "a pure negative" for the company, in part because "debating ideas is harder now."

The emphasis on vigorous debate at Netflix ... clarifies a truth that many approaches to strategy obscure: At their core, all great strategies are arguments....

Arguing is the best way to do strategy, especially in groups, provided the arguments follow established rules of engagement that are rooted in the principles of deductive logic. Great strategy demands the exchange and vetting of ideas - both in its development and implementation.

Listen to Patty McCord, former chief talent officer at Netflix, who asserted, "The main reason the company could continually reinvent itself and thrive, despite so many truly daunting challenges coming at us so fast and furiously, was that we taught people to ask, 'How do you know that's true?' Or my favorite variant, 'Can you help me understand what leads you to believe that's true?"' Such questions spawned vigorous internal debates at Netflix that, McCord said, "helped cultivate curiosity and respect and led to invaluable learning both within the team and among functions."

Jesper B. Sørensen and Glenn R. Carroll, "Why Good Arguments Make Better Strategy," Sloan Management Review 3 June 2021 https:// sloanreview.mit.edu/article/why-goodarguments-make-better-strategy/

\section{Technology and disruption}

\section{Building Google 2.0}

In 1998 a couple of Stanford graduate students published a paper describing a new kind of search engine: "In this paper, we present Google, a prototype of a large-scale search engine which makes heavy use of the structure present in hypertext. Google is designed to crawl and index the Web efficiently and produce much more satisfying search results than existing systems."

The key innovation was an algorithm called PageRank, which ranked search results by calculating how relevant they were to a user's query on the basis of their links to other pages on the web. On the back of PageRank, Google became the gateway to the internet, and Sergey Brin and Larry Page built one of the biggest companies in the world.

Now a team of Google researchers has published a proposal for a radical redesign that throws out the ranking 
approach and replaces it with a single large Al language model-a future version of BERT or GPT-3. The idea is that instead of searching for information in a vast list of web pages, users would ask questions and have a language model trained on those pages answer them directly. The approach could change not only how search engines work, but how we interact with them....

The problem is that even the best search engines today still respond with a list of documents that include the information asked for, not with the information itself. Search engines are also not good at responding to queries that require answers drawn from multiple sources. It's as if you asked your doctor for advice and received a list of articles to read instead of a straight answer.

Metzler and his colleagues are interested in a search engine that behaves like a human expert. It should produce answers in natural language, synthesized from more than one document and back up its answers with references to supporting evidence, as Wikipedia articles aim to do.

Will Douglas Heaven "The way we search online hasn't changed in decades. A new idea from Google researchers could make it more like talking to a human expert," MIT Technology Review 14 May 2021 www.technologyreview.com/2021/05/ 14/1024918/language-models-gpt3search-engine-google/

\section{Technologies that will drive disruption}

To identify the most disruptive trends, Bain met regularly with hundreds of carefully selected technology companies and startups. Combining those conversations with our experience from working with large corporations and our innovation ecosystem partners, we selected nine trends that have recently risen to the top of C-suite agendas.

1. Businesses "nudge" their customers toward desired behaviors.

Almost $60 \%$ of global executives across sectors said they adopted customer experience tools to improve different stages of the customer journey over the past five years.

\section{Gamification reskills the remote workforce.}

To make training more efficient and enjoyable, leading companies across industries such as retail, healthcare and professional services have turned to gamification.

\section{Diversity becomes a core recruiting metric for successful companies.}

In the past year, a racial reckoning prompted many companies to publicly commit to improving their diversity, equity and inclusion (DEI) agenda.

4. Brands and retailers turn into marketplaces to benefit from network effects.

Individual businesses have started moving away from these platforms to establish their own marketplaces. These owned marketplaces allow brands to benefit from the network effect: They can aggregate and sell adjacent products and services, creating a one-stop shop for their target customer.

\section{Integrated Business Planning (IBP) platforms optimize supply chains.}

IBP platforms are helping global companies bypass silos to transform their supply chains, revenue and financial planning and decisionmaking processes.
6. Al-enabled collective intelligence curates frontline insights for a more agile strategy.

According to a 2020 study by Harvard Business Review, $86 \%$ of executives say frontline workers need better technology-enabled insight to make good decisions in the moment.

\section{Savvy consumers demand sustainable products.}

When it comes to sustainability, consumers are paying far more attention and taking far more action. According to a 2018 Nielsen study, $73 \%$ of global consumers say they would definitely or probably change their consumption habits to reduce their impact on the environment.

\section{Quantum computing starts to} address specific applications.

With an estimated market size of \$1.1 billion in 2019 _ expected to grow five times by 2025-quantum computing is an emerging technology poised to radically reshape the world, by solving difficult problems beyond the power of any conventional supercomputer.

\section{NFTs remain the talk of the town.}

It's impossible to ignore the current buzz around NFTs. These digital identifiers attach verified ownership to virtual items in blockchain, protecting them from replication, making them identifiably unique, increasing their value and generating business opportunities.

Laurent-Pierre Baculard, Truc Mai Dupon Vohong and Vidur Khanna, "Nine Tech Innovation Trends Leading the Executive Agenda in 2021," Bain Insights 9 June 2021 www.bain.com/insights/nine-techinnovation-trends-leading-theexecutive-agenda-in-2021/

\section{Culture and innovation}

\section{Agility and competitive performance}

Is agility just noise from the IT department, or an opportunity that merits serious attention from the top 
team? And if pursuing agility yields benefits, what is the recipe for success?

To find the answers, we conducted a McKinsey Global Survey that reached 2,190 respondents across industries and geographies. We asked respondents what, if anything, their companies did in practice to advance agility and what hard numbers they achieved regarding business impact....

Agility at scale goes beyond adding more agile teams and team-level practices. The broader operating model, the connective tissue between and across the teams, also needs to be transformed. The organizations driving highly successful agile transformations made sure to do that by building an effective, stable backbone. This means optimizing the full operating model across strategy, structures, processes, people and technology by going after flat and fluid structures built around highperforming cross-functional teams, instituting more frequent prioritization and resource-allocation processes, building a culture that enables psychological safety and decoupling technology stacks.

Enterprise agility is thus a paradigm shift away from multilayered reporting structures, rigid annual budgeting, compliance-oriented culture, separation of business and technology, and other traits dominating organizations for the past hundred years....While individual case studies and agile success stories have been plentiful, having quantifiable results and a larger sample allowed us to go beyond anecdotes for the first time.

Agility results in a step change in performance and makes it possible to overtake born-agile organizations. Highly successful agile transformations typically delivered around 30 percent gains in efficiency, customer satisfaction, employee engagement and operational performance; made the organization five to ten times faster; and turbocharged innovation. While conventional wisdom sometimes sees these targets as contradictory (for example, efficiency at the cost of employee engagement), our results show otherwise. The respondents, on average, reported gains across four dimensions of performance, out of seven included in the survey.

This step change also showed up as a competitive advantage.

Organizations that achieved a highly successful agile transformation had a three times higher chance of becoming a top-quartile performer among peers than those who had not transformed. And they also overtook the born-agile organizations: they not only had a higher chance of becoming a top-quartile performer, but also had a greater chance of achieving a more mature operating model across all dimensions.

Wouter Aghina, Christopher Handscomb, Olli Salo and Shail Thaker, "The impact of agility: How to shape your organization to compete," McKinsey Quarterly 25 May 2021 www. mckinsey.com/business-functions/ organization/our-insights/the-impact-ofagility-how-to-shape-your-organizationto-compete

\section{Crisis and the paradox of innovation}

Starbucks was prescient in its pandemic strategy. The company responded to the upheaval that struck so many businesses last year by rapidly expanding its digital payment app to sign up millions of users. App customers contributed $50 \%$ of Starbucks' U.S. sales in the first quarter this year, which also saw revenue increase by $11 \%$ over last year.

It's a great example of what Wharton professor Serguei Netessine calls "innovate or perish." This descriptor is specific to businesses that are forced by a crisis to pivot on a dime.
"As we went into the pandemic, it became clear that in order to continue operating, you need to become increasingly digital," Netessine said, noting the pressure on retailers to respond to a massive surge in online shopping. "All those businesses could continue to operate, but they needed to have online capabilities, which many of them didn't have."

He reminded managers that innovating in times of crisis is easier for firms that maintain a forwardthinking mindset and a culture of experimentation. ...When a sudden disruption hits, there's no time or money to form and execute a survival plan from scratch. "Unfortunately, that's what most businesses do," Netessine said. "They innovate when it is a time of crisis."

"Innovate or Perish: What Businesses Should Learn from the Pandemic," Knowledge@Wharton7 June 2021

https://knowledge.wharton.upenn. edu/article/netessine-covid-businessinnovation/

\section{Business schools and the experimental revolution}

A combination of the availability of massive amounts of consumer data and improved computing power, plus the fact that it's easier and cheaper to randomize participants than ever before, has created the perfect conditions for the rise of what's being called the "experimental revolution" in business. As businesses increasingly demand actionable data to inform their decisions by leveraging experiments, MBA programs need to do more to equip future leaders with the necessary skills.

The value of experiments in nonscientific organizations is quite high. Instead of calling in managers to solve every puzzle or dispute large and small teams can run experiments and measure outcomes of interest and, armed with new data, decide for themselves, or at least put forward a 
proposal grounded in relevant information. The data also provide tangible deliverables to show to stakeholders to demonstrate progress and accountability....

But as more and more companies are embracing a culture of experimentation, they face a major challenge: talent. Experiments are difficult to do well. Some challenges include special statistical knowledge, clear problem definition and interpretation of the results. And it's not enough to have the skillset. Experiments should ideally be done iteratively, building on prior knowledge and working toward deeper understanding of the question at hand....

For example, General Mills recently hired for their innovator incubator group, called g-works, advertising for employees who are "using entrepreneurial skills and an experimental mindset" in what they called a "test and learn environment, with rapid experimentation to validate or invalidate assumptions."

Elizabeth R. Tenney, Elaine Costa and Ruchi M. Watson, "Why Business Schools Need to Teach Experimentation," Harvard Business Review 16 June 2021

https://hbr.org/2021/06/why-businessschools-need-to-teachexperimentation

\section{Agility and resilience in the wake of the pandemic}

In early 2021, Deloitte and Fortune surveyed CEOs about their leadership through the pandemic. Fully $85 \%$ indicated that their organizations had significantly accelerated digital transformation during the crisis, with three-quarters seeing the pandemic as fostering the formation of new partnerships and alliances....So things are changing rapidly, illustrating how moments of acute disruption can force consideration of how to better adapt to more chronic disruptions. And for many of Deloitte's clients, digital strategies are being guided by lessons from 2020:

Transform. The sudden need to accelerate digital customer solutions, workforce productivity and collaboration tools and global supply chain transformation has reframed what's possible, reset expectations around what's needed and revealed the choke points in an environment where businesses need to continue to adapt to change.

Focus. The pandemic highlighted the most important parts of the business, where leaders should focus strategies and how to execute transformation more effectively. At the same time, it has showed the value of innovation and co-creating more strategic opportunities for companies, their partners and their ecosystems.

Execute. The crisis has shown the need for more resilient operations and infrastructure to future-proof the business, enhance security and enable financial performance, not only for resiliency but as a pathway to higher performance. Deloitte's 2020 digital transformation survey has uncovered a link between digital maturity and financial performance....

While the imperative to transform may be clear, the path is challenging. CEOs are pressed to prioritize digital solutions that meet the needs of the present while building a platform for innovation and competitive advantage. This can require changes in processes and infrastructure, such as shifting workloads to the cloud, deploying artificial intelligence (AI) across the enterprise and reevaluating how, where and by whom work is done. For some, it may include transforming network capabilities and deploying edge computing infrastructure. Such change may demand new, continuously evolving workforce skills in an environment where diversity, equity and inclusion are highly valued.
Janet Foutty, "How digital transformation-and a challenging environment-are building agility and resilience," Deloitte Insights 29 April 2021 Areas to accelerate digital transformation | Deloitte Insights

\section{A wider view}

\section{Understanding how disasters happen}

A remarkably common issue is complacency. Organizations tend to drift to a higher risk state, relaxing safeguards and controls as they try to accommodate conflicting business goals and tradeoffs. If nothing happens for long stretches of time the view becomes that nothing will continue to happen in the future.... Krausmann refers to a disaster "incubation" period, where a system moves closer to the edge of its safety envelope until it fails.

There are also difficulties with retaining information from previous incidents, poor knowledge sharing, failure to use available knowledge, corporate memory loss due to changes in staff and management, frequent ownership changes and instability in business continuity.... Similarly, rare events with complex causal relationships are hard to grasp. A range of cognitive biases explain why events may be referred to as Black Swans:

- Confirmation bias: we look for evidence that confirms our beliefs but ignore facts that would refute them.

- Narrative fallacy: we construct simplified stories out of sequential facts to make sense of the world.

- Silent evidence: a sampling bias where only evidence that catches the eye is considered rather than searching for and considering what is also there. 
- Ludic fallacy: attempts to predict the future with tools and models that cannot capture rare events. Mathematical models of an uncertain risk create a false sense of certainty, thereby possibly doing more harm than good. "No probabilistic model based on in-box thinking can deal with out-of-box type events."
In complex and tightly-coupled systems, small initial shocks can propagate through the individual subsystems, interacting in unexpected ways and creating a chain reaction that can ultimately lead to complete system failure.

"Thinking the unthinkable" SAMI

Consulting, 10 June 2021 https:// samiconsulting.co.uk/thinking-theunthinkable/

\section{Corresponding author}

Craig Henry can be contacted at: craig_henry@centurylink.net 